Теорія Ймовір. та Матем. Статист. Вип. 78, 2008
Theor. Probability and Math. Statist.

No. 78, 2009, Pages 15-21 S 0094-9000(09)00758-3

Article electronically published on August 4, 2009

\title{
ANALYTICAL PROBLEMS OF THE ASYMPTOTIC BEHAVIOR OF MARKOV FUNCTIONALS. II
}

UDC 519.21

\author{
S. V. DEGTYAR'
}

Abstract. The asymptotic behavior of Markov functionals of a homogeneous ergodic Markov process is studied in this paper.

This paper is a continuation of [1]. We number the displayed formula continuously after the last number in [1].

Let us turn to the proof of the theorem. First we show that

$$
h_{\varepsilon}^{i}(x, t \cdot s) \underset{\substack{\varepsilon \rightarrow 0 \\ t \rightarrow \infty \\ \varepsilon t \rightarrow u}}{\longrightarrow} \delta^{i l} e^{c_{i i} u s}\langle\pi, \varphi\rangle .
$$

We recall that $\langle\pi, \varphi\rangle=\int_{E} \pi(d y) \varphi(y)$ and the convergence is uniform with respect to $x \in D$ and $t \in[\sigma, T]$ for all $T>\sigma>0$.

To prove the latter relation we use a result similar to the Markov renewal theorem. Let a family of nonnegative semihomogeneous kernels $G_{\varepsilon}(x, d y \times d t)$ depending on a small parameter $\varepsilon>0$ converge as $\varepsilon \rightarrow 0$ to a nonnegative stochastic kernel $G(x, d y \times d t)$ in the sense that

$$
\sup _{A \in \mathcal{A}}\left\|\int_{0}^{\infty} G_{\varepsilon}(\cdot, A \times d t) \varphi(t)-\int_{0}^{\infty} G(\cdot, A \times d t) \varphi(t)\right\| \underset{\varepsilon \rightarrow 0}{\longrightarrow} 0
$$

for all continuous bounded functions $\varphi(t), t \geq 0$, where

$$
\|f\|=\inf \{c:|f(x)| \leq \operatorname{ch}(x)\} \quad \pi \text {-almost everywhere. }
$$

Denote by $G_{\varepsilon}(x, d y)$ and $G(x, d y)$ the bases of the kernels $G_{\varepsilon}(x, d y \times d t)$ and $G(x, d y \times d t)$, respectively. Let $U_{\varepsilon}(x, d y \times d t)$ denote the potential of the kernel $G_{\varepsilon}(x, d y \times d t)$.

We assume that the kernel $G(x, A)$ does not depend on $x$, that is,

$$
G(x, A)=\pi(A) \quad \text { and } \quad h(x)=1,
$$

where $\pi(A)$ is a probability distribution on $(E, \mathcal{B})$.

We denote by $\lambda_{\varepsilon}$ the spectral radius of the kernel $G_{\varepsilon}(x, A)=G_{\varepsilon}(x, A \times[0, \infty))$ and by $\pi_{\varepsilon}$ the eigenprobability measure of the kernel $G_{\varepsilon}(x, A)$ corresponding to the eigenvalue $\lambda_{\varepsilon}$. Put $\gamma_{\varepsilon}=\left(1-\lambda_{\varepsilon}\right) / m$.

2000 Mathematics Subject Classification. Primary 60K15; Secondary 60J25.

Key words and phrases. Markov functionals, homogeneous ergodic process. 
Theorem 1. Assume that conditions (49) and (50) hold. We also assume that

a) the kernel $G(x, d y \times d t)$ is nonlattice and

$$
\begin{gathered}
\sup _{\varepsilon>0} \sup _{x \in E} \int_{0}^{\infty} G_{\varepsilon}(x, E \times d t) t<\infty, \\
\sup _{\varepsilon>0} \int_{E} \int_{T}^{\infty} \pi(d x) G_{\varepsilon}(x, E \times d t) t \underset{T \rightarrow \infty}{\longrightarrow} 0 ;
\end{gathered}
$$

b) a family of $\mathcal{B} \times \mathcal{B}_{+}$-measurable functions $g_{\varepsilon}(x, t)$ is such that $g_{\varepsilon}(x, t) \geq 0$ and

$$
\begin{gathered}
\sup _{\varepsilon>0} \sup _{x \in E} \int_{0}^{\infty} g_{\varepsilon}(x, t) d t<\infty, \\
\sup _{\varepsilon>0} \sup _{x \in E} \sup _{t \geq 0} U_{\varepsilon} * g_{\varepsilon}(x, t)<\infty, \\
\lim _{N \rightarrow \infty} \sup _{\varepsilon>0} \sup _{x \in E} \sum_{k=N}^{\infty} \sup _{k \leq t \leq k+1} g_{\varepsilon}(x, t)=0, \\
\sup _{\varepsilon>0} \delta \int_{E} \pi(d x) \sum_{k=0}^{\infty}\left\{\sup _{k \delta \leq t \leq k \delta+\delta} g_{\varepsilon}(x, t)-\inf _{k \delta \leq t \leq k \delta+\delta} g_{\varepsilon}(x, t)\right\} \underset{\delta \rightarrow 0}{\longrightarrow} 0 ;
\end{gathered}
$$

c) there exists a $\mathcal{B}_{+}$-measurable function $g(t)$ such that

$$
\int_{0}^{\infty}\left|g(t)-\int_{E} \pi(d x) g_{\varepsilon}(x, t)\right| d t \underset{\varepsilon \rightarrow 0}{\longrightarrow} 0 ;
$$

d) there exists a number $\delta>0$ such that

$$
\begin{gathered}
\inf _{\varepsilon>0} \inf _{x \in E} G_{\varepsilon}(x, E \times[\delta, \infty)) \geq \delta, \\
\int_{E} \pi_{\varepsilon}(d x) G_{\varepsilon}(x, A \times[\delta, \infty)) \geq \delta \pi_{\varepsilon}(A) .
\end{gathered}
$$

Then

$$
\lim _{\substack{\gamma_{\varepsilon} t \rightarrow c \\ \varepsilon \rightarrow 0 \\ t \rightarrow \infty}} U_{\varepsilon} \times g_{\varepsilon}(x, t)=e^{-c} \frac{1}{m} \int_{0}^{\infty} g(s) d s
$$

uniformly with respect to $x \in E$, where

$$
m=\int_{E} \int_{0}^{\infty} \pi(d x) G(x, E \times d t) t<\infty .
$$

Conditions (12)-(15) imply that the kernels $Q_{\varepsilon}^{i}$ satisfy all the assumptions of Theorem 2. Now we show that the functions $g_{\varepsilon}^{i}(x, t)$ satisfy conditions $(55)-(57)$. Without loss of generality, we assume that $0 \leq \varphi(x) \leq 1$. Then

$$
\begin{aligned}
\sup _{k \leq t \leq k+1} g_{\varepsilon}^{i}(x, t) & =\sup _{k \leq t \leq k+1} \mathbb{P}_{x, i}\left\{\varphi(X(t)), \xi_{\varepsilon}(t)=l, t \leq \tau\right\} \leq \mathbb{P}_{x}\{k<\tau\} \\
& \leq \int_{k-1}^{k} \mathbb{P}_{x}\{\tau>t\} d t .
\end{aligned}
$$

Thus

$$
\begin{aligned}
\sup _{x \in D} \sum_{k=N+1}^{\infty} \sup _{k \leq t \leq k+1} g_{\varepsilon}^{i}(x, t) & =\sup _{x \in D} \sum_{k=N+1}^{\infty} \int_{k-1}^{k} \mathbb{P}_{x}\{\tau>t\} d t=\sup _{x \in D} \int_{N}^{\infty} \mathbb{P}_{x}\{\tau>t\} d t \\
& \leq \sup _{x \in D}\left[\mathbb{P}_{x}\{\tau>N\}+\mathbb{P}_{x}\{\tau, \tau>N\}\right] .
\end{aligned}
$$


In view of (14), the latter expression approaches zero as $N \rightarrow \infty$, and this proves the uniform convergence of series (55) with respect to $n \geq 1$ and $x \in D$.

Now we check condition (56). It follows from (16) that the process $\left\{\varphi(X(t)), \xi_{\varepsilon}(t)\right\}$ is stochastically continuous uniformly in $\varepsilon>0$ and $t \in[0, T]$ for an arbitrary initial distribution of the process $X(t)$. In particular,

$$
\begin{aligned}
& \sup _{a \leq s \leq t \leq b} \mathbb{P}_{x, i}|\varphi(X(t))-\varphi(X(s))| \underset{\substack{b-a \rightarrow 0 \\
0 \leq a \leq b \leq T}}{\longrightarrow} 0, \\
& \sup _{a \leq s \leq t \leq b} \sup _{\varepsilon>0} \mathbb{P}_{x, i}\left\{\xi_{\varepsilon}(t)=l, \xi_{\varepsilon}(s) \neq l\right\} \underset{\substack{b-a \rightarrow 0 \\
0 \leq a \leq b \leq T}}{\longrightarrow} 0
\end{aligned}
$$

for all $T>0, i, l \in I$, and $x \in E$.

Next we apply the inequality

$$
\begin{aligned}
\sup _{a \leq t \leq b} & g_{\varepsilon}^{i}(x, t)-\inf _{a \leq t \leq b} g_{\varepsilon}^{i}(x, t) \\
\leq & \sup _{a \leq s \leq t \leq b} \mathbb{P}_{x, i}|\varphi(X(t))-\varphi(X(s))|+\sup _{a \leq s \leq t \leq b} \mathbb{P}_{x, i}\left\{\xi_{\varepsilon}(t)=l, \xi_{\varepsilon}(s) \neq l\right\} \\
& +\mathbb{P}_{x, i}\{a \leq \tau \leq b\} .
\end{aligned}
$$

The further reasoning is the same as that used in the proof of Theorem 3, Chapter 2, Section 2 in [2] and this proves condition (56). Since

$$
\lim _{\varepsilon \rightarrow 0} \mathbb{P}_{x, i}\left\{\xi_{\varepsilon}(t) \neq i\right\}=0,
$$

relation (57) follows from (13) for all $x \in E, i \in I, t \geq 0$, and for the function

$$
g(t)=\delta^{i l} \mathbb{P}_{\pi_{D}}[\varphi(X(t)), t<\tau] .
$$

Note that

$$
\frac{1}{m} \int_{0}^{\infty} g(t) d t=\delta^{i l}\langle\pi, \varphi\rangle
$$

whence (48) follows.

Consider a sequence of functions $p_{k}^{i}(t), k \geq 0$, defined by the following recurrence relation:

$$
\begin{gathered}
p_{0}^{i}(t)=\delta^{i l} e^{c_{i i} t}, \\
p_{k+1}^{i}(t)=\int_{0}^{t} e^{c_{i i} s} d s \sum_{j \neq i} c_{i j} p_{k}^{j}(t-s) .
\end{gathered}
$$

Note that the sum of the series

$$
\sum_{k=0}^{\infty} p_{k}^{i}(t)=p^{i l}(t)
$$

is equal to the entry $(i, l)$ of the matrix $\exp \{t C\}$, where

$$
C=\left\|c_{i j}\right\|_{i, j=1}^{\infty} .
$$

We prove by induction in $k \geq 0$ that

$$
\lim _{\substack{\varepsilon \rightarrow 0 \\ t \rightarrow \rightarrow \infty \\ \varepsilon t \rightarrow u}} h_{\varepsilon, k}^{i}(x, t)=p_{k}^{i}(u)\langle\pi, \varphi\rangle
$$

for all $u \geq 0$ and $i \in I$ uniformly in $x \in D$. Recall that the functions $h_{\varepsilon, k}^{i}(x, t)$ are defined in (19). 
Equality (61) coincides with (48) for $k=0$. We show that (48) holds for $k=1$ uniformly in $0 \leq t \leq T$ and $x \in D$ for all $T>0$. We have

$$
h_{\varepsilon, 1}^{i}(x, t)=\sum_{j \neq i} \int_{D} \int_{0}^{1} R_{\varepsilon}^{i j}(x, d y \times t d s) h_{\varepsilon}^{j}(y, t(1-s)) .
$$

Using (48) we get

$$
h_{\varepsilon}^{j}(y, t(1-s))=\langle\pi, \varphi\rangle p_{0}^{j}(u(1-s))+r_{\varepsilon}^{j}(y(1-s)),
$$

where

$$
\sup _{y \in D} \sup _{0 \leq t \leq T} \sup _{0 \leq s \leq T-\sigma}\left|r_{\varepsilon}^{j}(y, 1-s)\right| \underset{\varepsilon \rightarrow 0}{\longrightarrow} 0 .
$$

The term corresponding to an index $j$ on the right hand side of (62) can be represented as follows:

$$
\begin{aligned}
\int_{0}^{1} & R_{\varepsilon}^{i j}(x, D \times t d s) p_{0}^{j}(u(1-s))\langle\pi, \varphi\rangle+\int_{D} \int_{0}^{1-\sigma} R_{\varepsilon}^{i j}(x, d y \times t d s) r_{\varepsilon}^{j}(y, 1-s) \\
& +\int_{D} \int_{1-\sigma}^{1} R_{\varepsilon}^{i j}(x, d y \times t d s) r_{\varepsilon}^{j}(y, 1-s) .
\end{aligned}
$$

According to Lemma 2, the first term of (64) converges as $\varepsilon \rightarrow 0$ to $\langle\pi, \varphi\rangle p_{1}^{j}(u)$ uniformly in $x \in D$ and $0 \leq u \leq T$. We apply Lemma 2 to the second term and deduce from (63) that it converges to zero uniformly in $x \in D$ and $0 \leq u \leq T$. If $\sigma>0$ is sufficiently small, then the third term also is small uniformly in $x \in D$ and $0 \leq u \leq T$.

The proof of the induction step from $k$ to $k+1$ for $k \geq 1$ is the same as above, and thus we omit it.

It follows from (20), (21), (61), (17), and (48) that

$$
\lim _{\substack{\varepsilon \rightarrow 0 \\ t \rightarrow \infty \\ \varepsilon t \rightarrow u}} f_{\varepsilon}^{i}(x, t)=p^{i l}(u)\langle\pi, \varphi\rangle
$$

for all $x \in E$ and $u>0$. Thus the theorem is proved if (18) holds.

To complete the proof of the theorem we need to check condition (18). It is easy to see that the functions

$$
\tilde{f}_{\varepsilon}^{i}(x, t)=e^{-\varepsilon t} f_{\varepsilon}^{i}(x, t)
$$

satisfy the system of equations similar to (16); namely,

$$
\widetilde{f}_{\varepsilon}^{i}(x, t)=\widetilde{g}_{\varepsilon}^{i}(x, t)+\widetilde{Q}_{\varepsilon}^{i} * \widetilde{f}_{\varepsilon}^{i}(x, t)+\sum_{j \neq i} \widetilde{Q}_{\varepsilon}^{i j} * \widetilde{f}_{\varepsilon}^{j}(x, t),
$$

where

$$
\begin{aligned}
\widetilde{g}_{\varepsilon}^{i}(x, t) & =e^{-\varepsilon t} g_{\varepsilon}^{i}(x, t), \\
\widetilde{Q}_{\varepsilon}^{i}(x, d y \times d t) & =e^{-\varepsilon t} Q_{\varepsilon}^{i}(x, d y \times d t), \\
\widetilde{Q}_{\varepsilon}^{i j}(x, d y \times d t) & =e^{-\varepsilon t} Q_{\varepsilon}^{i j}(x, d y \times d t) .
\end{aligned}
$$

Following the method used in the first part of the paper to derive (17) from (16) we obtain from representation (64) that

$$
\widetilde{f}_{\varepsilon}^{i}(x, t)=\widetilde{H}_{\varepsilon}^{i} * g_{\varepsilon}^{i}(x, t)+\sum_{j \neq i} \widetilde{R}_{\varepsilon}^{i j} * \widetilde{f}_{\varepsilon}^{j}(x, t),
$$

where $\widetilde{H}_{\varepsilon}^{i}$ is the potential of the kernel $Q_{\varepsilon}^{i}$,

$$
\widetilde{R}_{\varepsilon}^{i j}=\widetilde{H}_{\varepsilon}^{i} * \widetilde{Q}_{\varepsilon}^{i j} .
$$


Now we check that the kernels $\widetilde{R}_{\varepsilon}^{i j}(x, d y \times d t)$ satisfy condition (18). Recalling the notation for $\widehat{R}_{\varepsilon, \alpha}^{i j}(x, A)$ we obtain

$$
\widetilde{R}_{\varepsilon}^{i j}(x, A \times[0, \infty))=\widetilde{R}_{\varepsilon, 1}^{i j}(x, A), \quad A \in \mathcal{B} .
$$

This together with (41) and Lemma 1 yields

$$
\sup _{x \in D} \sum_{j \neq i} \widetilde{R}_{\varepsilon}^{i j}(x, D \times[0, \infty)) \leq \frac{-c_{i i}}{1-c_{i i}}
$$

for all sufficiently small $\varepsilon>0$. Putting

$$
r=\sup _{i} \frac{-u c_{i i}}{1-u c_{i i}}
$$

we prove condition (18) with $\widetilde{R}_{\varepsilon}^{i j}$ instead of $R_{\varepsilon}^{i j}$.

Assuming that conditions (17)-(19) and (47) hold, we get

$$
\lim _{\varepsilon \rightarrow 0} \frac{1}{\varepsilon m}\left[\mathbb{P}_{\pi_{D}, i}\left\{e^{-\varepsilon \tau}, \xi_{\varepsilon}(\tau)=i\right\}-1\right]=c_{i i}-1 .
$$

Applying the part of Theorem 1 already proved to the function $\widetilde{f}_{\varepsilon}^{i}(x, t)$, we find that

$$
\lim _{\substack{\varepsilon \rightarrow 0 \\ t \rightarrow \infty \\ \varepsilon t \rightarrow u}} \widetilde{f}_{\varepsilon}^{i}(x, t)=p^{i l}(u)\langle\pi, \varphi\rangle
$$

if relations (17)-(19) and (47) hold, where

$$
\left\|p^{i l}(u)\right\|_{i, l=1}^{\infty}=\exp \{u(C-I)\}=e^{-u} \exp \{u C\} .
$$

On the other hand,

$$
\tilde{f}_{\varepsilon}^{i}(x, t)=e^{-\varepsilon t} f_{\varepsilon}^{i}(x, t) .
$$

Comparing this result with equality (66) we complete the proof of the theorem in the general case.

The proof of Theorem 4 follows the same lines; thus we omit it. In the proof one should apply the following result.

Theorem 2. Assume that conditions (49)-(51) hold and

a) the step of the kernel $G(x, d y \times d t)$ is equal to one and

$$
G_{\varepsilon}(x, E \times[0, \infty))=\sum_{k=0}^{\infty} G_{\varepsilon}(x, E \times\{k\})
$$

for all $x \in E$ and $n \geq 1$;

b) $\mathcal{B}$-measurable functions $g_{\varepsilon}(x, k), \varepsilon>0, k \geq 1$, are such that

$$
\sup _{\varepsilon>0} \sup _{x \in E} \sum_{k \geq N} g_{\varepsilon}(x, k) \underset{N \rightarrow \infty}{\longrightarrow} 0
$$

c) there exists a sequence $g_{k}$ such that

$$
\sum_{k \geq 0}\left|g_{k}-\int_{E} \pi(d y) g_{\varepsilon}(x, k)\right| \underset{\varepsilon \rightarrow 0}{\longrightarrow} 0 .
$$

Then

$$
\lim _{\substack{\varepsilon \rightarrow 0 \\ k \rightarrow \infty \\ \gamma_{\varepsilon} \rightarrow \infty}} U_{\varepsilon} * g_{\varepsilon}(x, k)=e^{-c} \frac{1}{m} \sum_{k \geq 0} g_{k} .
$$


Theorem 3. Assume conditions (1), (2), (11), and (17)-(19). Let the parameter $t$ vary in the set $\{0,1, \ldots\}$.

Then

$$
\left\{\mathbb{P}_{x, i}\left[\varphi(X(t)), \xi_{\varepsilon}(t)=j\right]-p_{i j}(u) \int_{E} \pi(d y) \varphi(y)\right\} \underset{\substack{\varepsilon \rightarrow 0 \\ t \rightarrow \infty \\ \varepsilon t \rightarrow u}}{\longrightarrow \rightarrow} 0
$$

for all $u \geq 0, i, j \in I, x \in E$, and all $\mathcal{B}$-measurable bounded functions $\varphi(y)$, where $p_{i j}(u)$ is the entry $(i, j)$ of the matrix $e^{u C}, C=\left\|c_{i j}\right\|_{i, j=1}^{\infty}$.

\section{Concluding Remarks}

Remark 1. Condition (16) holds if

$$
\sup _{\varepsilon>0} \sup _{x \in E} \mathbb{P}_{x, i}\left\{\xi_{\varepsilon}(t) \neq i\right\} \underset{t \rightarrow 0}{\longrightarrow} 0
$$

for all $i \in I$.

Remark 2. We assume condition (16) in order to apply the theorem to the functions $g_{\varepsilon}^{i}(x, t)$. Let $\Psi(y, j)$ be a bounded function of two arguments $y \in E$ and $j \in I$ that is continuous in $y \in E$ for all $i \in I$. Then the functions

$$
g_{\varepsilon}^{i}(x, t)=\mathbb{P}_{x, i}\left\{\Psi\left(X(t), \xi_{\varepsilon}(t)\right), t<\tau\right\}=\mathbb{P}_{x, i}\left\{\Psi\left(X(t), \xi_{\varepsilon}(t)\right), t<\left(\tau \wedge \zeta_{\varepsilon}\right)\right\}
$$

satisfy the assumptions of the theorem if the integer-valued stochastic process

$$
\Psi\left(X(t), \xi_{\varepsilon}(t)\right), \quad t \geq 0,
$$

is stochastically continuous uniformly in $\varepsilon>0$, that is, if

$$
\sup _{\varepsilon>0} \mathbb{P}_{x, i}\left|\Psi\left(X(t), \xi_{\varepsilon}(t)\right)-\Psi\left(X(s), \xi_{\varepsilon}(s)\right)\right| \underset{s \rightarrow t}{\longrightarrow} 0
$$

for all $x \in E, i \in I$, and $t \geq 0$.

This remark implies the following result.

Corollary 1. If conditions (2) and (67) hold, then

$$
\lim _{\substack{\varepsilon \rightarrow 0 \\ t \rightarrow \infty \\ \varepsilon t \rightarrow u}}\left\{\mathbb{P}_{x, i} \Psi\left(X(t), \xi_{\varepsilon}(t)\right)-\sum_{j=1}^{\infty} p_{i j}(u) \int_{E} \Psi(y, j) \pi(d y)\right\}=0
$$

for all $x \in E, i \in I$, and $u \geq 0$.

Setting $\Psi(y, j) \equiv 1$ we obtain the following result.

Corollary 2. If condition (2) holds, then

$$
\left.\lim _{\substack{\varepsilon \rightarrow 0 \\ t \rightarrow \infty \\ \varepsilon t \rightarrow u}} \mathbb{P}_{x, i}\left\{\varepsilon \zeta_{\varepsilon} \geq u\right\}-\sum_{j=1}^{\infty} p_{i j}(u)\right\}=0
$$

for all $x \in E, i \in I$, and $u>0$.

\section{BIBLIOGRAPHY}

1. S. V. Degtyar', Analytical problems of the asymptotic behavior of Markov functionals. I, Teor. Imovir. Matem. Statist. 77 (2007), 28-35; English transl. in Theory Probab. Math. Statist. 77 (2008), 31-38. MR2432770

2. V. M. Shurenkov, Ergodic Theorems and Related Problems, Nauka, Moscow, 1989; English transl., VSP International Science Publishers, Leiden, 1998. MR1690361 (2000i:60002) 
3. N. I. Portenko, A. S. Skorokhod, and V. M. Shurenkov, Markov Processes, Progress in Science and Technology. Current Problems in Mathematics. Fundamental Directions, vol. 46, Vsesoyuz. Inst. Nauchn. i Tekhn. Inform., Akad. Nauk SSSR, Moscow, 1989. (Russian) MR1039619 (91j:60113b)

4. T. Kato, Perturbation Theory for Linear Operators, Springer-Verlag, Berlin-New York, 1966. MR0203473 (34:3324)

Department of Higher Mathematics, Vadym Hetman Kyiv National Economic University, Peremogy Avenue 54/1, Kyiv 03057, Ukraine

E-mail address: sdegtyar@zra.kiev.ua

Received 6/MAY/2005

Translated by O. I. KLESOV 